\title{
Time to champion global data
}

Environmental threats makes the need for enhanced atmospheric monitoring obvious, but the will and organization to fund it are insufficient. Less obviously, global monitoring of fundamental geophysical parameters would bring great benefits.

Most busy people have on their desks problems that somehow never reach the top of the pile. The time required to deal with them might be short, yet somehow there is always something more urgent (or -let's be honest - more interesting) to get on with.

In Nature's offices, of course, this rare phenomenon tends to be restricted to internal memoranda. More interestingly, and more worryingly, something rather similar is a persistent problem in the geosciences. Nobody can quite get round to meeting one fundamental need: adequately and consistently recording, year on year, streams of data that may be individually prosaic but which cumulatively provide many of the best indicators of how our planet is constructed and is behaving. Geophysics and geochemistry (in their broadest senses, encompassing the solid Earth, the hydrosphere and the atmosphere) are much the worse for this chronic failure.

Occasionally a community gathers itself for a monumental effort. A notable early example was the International Geophysical Year (IGY), conceived by James van Allen and others in 1950, and implemented in 1957-58 with the participation of 67 countries. As it happened, during the IGY the first satellites were launched and nuclear weapons were exploded at high altitudes: related civil and military research both led to considerable new understanding of the Earth's magnetic and outer atmospheric environment. But the sheer global reach of observation was also a significant achievement. The International Geosphere-Biosphere Programme and the World Ocean Circulation Experiment are two of its successors, both established with quite specific research goals of global scope - respectively investigating geochemical cycles and fluxes between ocean basins.

There are other phenomena that need to be monitored in a less goal-directed spirit but no less purposefully. Take atmospheric aerosols. Their main sources at the surface and in the lower atmosphere are known, as are their important roles in radiative transfer (and hence climate) and in chemistry (and hence pollution). But details of those roles are inadequately understood, as are their temporal and spatial behaviour. As Meinrat Andreae, an atmospheric chemist, emphasized in last week's issue (Nature 380, 389-390; 1996), because of a lack of systematic measurements we cannot quantify the contribution from human activities over past decades - a significant lacuna in atmospheric modelling, and one that is likely to persist.

Again, the problem is a world-wide one. The US National Oceanic and Atmospheric Administration maintains a few monitoring stations, and the World Meteorological Organisation (WMO) is also making a significant contribution. There are field projects to examine particular aspects of aerosol behaviour - for example, coordinated measurements by satellite, airborne and ground-based experiments are being conducted this summer on the US East Coast to examine the radiative effects of sulphate aerosols. But the lower-profile long-term monitoring surely deserves greater support than it is getting.

The significant variability of aerosol distributions means that relatively dense monitoring networks are desirable. But other scientifically important phenomena suffer from overdense observations in some parts of the globe and a paucity elsewhere. This problem has been highlighted in a paper from the Geophysical Research Centre in Potsdam, Germany (I. I. Mueller et al., submit- ted to the Journal of Geodynamics). It usefully summarizes the conceptual links between a variety of geophysical parameters that each require global monitoring: gravity, surface and internal deformations of the Earth, dynamic interactions between the Earth, oceans and atmosphere, the planet's rotation, not to mention magnetic fields. Techniques being applied include satellite laser ranging, very-long-baseline interferometry, the Global Positioning System, seismology, tide and ground-water gauges, and superconducting gravimeters.

A glance at a global map of the distribution of such monitoring activities reveals unsurprising but problematic variations: concentrations in Europe and the United States and voids in less developed countries and in the vast expanse of the Pacific Ocean. Yet, as the authors emphasize, optimizing the distributions of the various networks would enhance by an order of magnitude the measurement precision of tectonic plate velocities and gravity parameters, for example. It would also open to experiment, among several processes, the relationship between ocean topography, ocean dynamics and the gravitational potential, as well as motions in the Earth's core detectable by variations of its rotation vector.

The organization that looks after such challenges is the International Union of Geodesy and Geophysics (IUGG). It has recently begun to look sympathetically at the issue, but there are obstacles. Possessiveness may be one. Which of the eight institutions in Western Europe with superconducting gravimeters is going to be sufficiently internationally minded to have its gravimeter sent off to somewhere in the Southern Hemisphere - which in its entirety hosts just one? And who would pay for maintenance and data processing at the new sites? That is a question of funding capacity but also of principle - should one country, comparatively well resourced but hard pressed, support a scientific facility based in another? Only if the science is sufficiently high up the list of the donor's national priorities - which seems questionable when it comes to a pricy monitoring station that forms but one element of a multinational network. And there will also be a tab to be picked up by the recipients.

Regrettably, the need to build (or redistribute) global networks comes at a time when the budgetary environment is rapidly worsening. Two years ago, for example, the US National Aeronautics and Space Administration (NASA) was operating a set of six laserranging stations at a cost of $\$ 6$ million a year. Now that budget has collapsed, and NASA is seeking to dispose of the equipment to anybody who might usefully take it on, also offering just one year of technical support - Tahiti is one possible new location.

Lacking the immediate environmental relevance of aerosols, and potentially involving substantial international transfers of technological resources that amount to a form of foreign aid, the task of filling those observational gaps presents the geophysical community with something of a political challenge, to put it mildly. Without the recent backing of the IUGG, there would have been no hope of progress. A modest target is that, three years down the road, a well-distributed network of 10 multi-instrumented geophysical reference stations might be in operation. The scientific benefits are clear, but whether some coalition of agencies can muster sufficient resolve to achieve even that development remains questionable. That is a sorry state of affairs. 